\title{
Percepción del estado nutricional y del estado nutricional real en escolares
}

\section{Clara Isabel Tejada Garrido, María Estela Colado Tello, Ana Palacios Ramos, Carmen Urbina Bravo, y Lourdes Basurto Sáenz Hospital San Pedro (España)}

\begin{abstract}
La obesidad infantil constituye el trastorno nutricional de mayor relevancia en nuestra sociedad y un grave problema sanitario. Diferentes investigaciones han analizado cómo el entorno distorsiona la percepción y subestima este problema. Los objetivos de este estudio son evaluar en escolares de 7 y 8 años la percepción de su estado nutricional mediante imágenes y medidas objetivas, evaluar su percepción de salud y enfermedad y evaluar la satisfacción con su imagen corporal. Se ha realizado un estudio descriptivo, transversal con 374 escolares pertenecientes a nueve centros educativos. Se recogieron datos de peso, talla, y se les pasó el test de imágenes de Warschburger Kröller. La mayoría infravalora su peso eligiendo figuras de categorías inferiores a la suya. En relación con la satisfacción con su imagen corporal los escolares están satisfechos con ella aunque se detecta una relación inversa entre el valor de percentil y el grado de satisfacción con su imagen. En general, los escolares perciben su salud como buena o muy buena.
\end{abstract}

Palabras clave: Escolares, índice de masa corporal, obesidad, percepción.

Perception of the nutritional status and real nutritional status of school children. Childhood obesity is the most relevant nutritional disorder of our society and a serious health problem. Several studies have analyzed how environment distorts society's perception and underestimates this problem. Several aims were evaluated in this study including; Nutritional status perception of 7- and 8-years old children using pictures and objective measurements, their perception of health and disease; and their level of satisfaction of their body image. A cross-sectional study was conducted on 374 children from 9 different schools. Weight and size data were collected and the Warschuburger Kröller test performed. We disclose that the majority of children underestimate their weight and they choose images from lower categories, associated with improved body satisfaction. Although an inverse relationship is detected between the value of the percentile and the level of satisfaction, the children are satisfied with their body image. In general, the children consider their health as good-very good.

Keywords: Schoolchild, body mass index (BMI), obesity, perception.

Correspondencia Clara Isabel Tejada Garrido. Avenida de la Paz 36 5'Izqda. C.P.: 26004 Logroño, La Rioja (España). E-mail: ctejada@riojasalud.es 
En los países industrializados, las deficiencias nutricionales graves en la infancia y la adolescencia prácticamente han desaparecido. Sin embargo, en el curso de los últimos años se ha incrementado progresivamente la prevalencia de la obesidad infantil y juvenil, constituyendo el trastorno nutricional de mayor relevancia en nuestro medio y un problema sanitario de gran trascendencia, especialmente si se tiene en cuenta que, la mayoría de los niños con sobrepeso u obesidad lo seguirán siendo en la adolescencia y en la edad adulta, con el riesgo sobreañadido de una mayor morbimortalidad (Durá y Sánchez-Valverde, 2005).

Según la Organización Mundial de la Salud (OMS) en el año 2016 había en el mundo más de 340 millones de niños y adolescentes, entre 5 y 19 años con sobrepeso u obesidad. En España, el informe Aladino realizado entre 2015 y 2016 a niños y niñas entre 6 y 11 años, concluye que la prevalencia de sobrepeso en nuestro país es de $23.2 \%$ y de obesidad del $18.1 \%$, utilizando los estándares de crecimiento de la OMS (2016). Si comparamos estos resultados con el estudio Aladino llevado a cabo en el año 2013, vemos que se ha producido una disminución estadísticamente significativa en la prevalencia de sobrepeso en niños y niñas entre 6 y 11 años (26.2\%); mientras que la prevalencia de la obesidad se halla estabilizada tanto en niños como en niñas (18.3\%).

Otras encuestas nutricionales que se han publicado en nuestro país, como el estudio EnKid (Serra, 2003) o la Encuesta Nacional de Salud de 2012, muestran la importancia, de primer orden, que este problema de salud pública tiene en nuestra sociedad.

La relevancia del sobrepeso y obesidad infantil no solo radica en el aumento de peso, sino además en la infinidad de patologías que se derivan de ella tanto física como psicológicas, así como los problemas de connotación social y de salud pública que comportan (Muñoz, Mazure, y Culebra, 2004). Entre las consecuencias más importantes de la obesidad se encuentran el aumento de la morbilidad y mortalidad del individuo, lo que se traduce en una menor esperanza y calidad de vida. Aunque suene alarmante y alarmista, ya se habla de generaciones que van a vivir menos que sus progenitores debido, entre otras causas, a un exceso de peso y sus consecuencias para la salud.

Se estima que más de un $40 \%$ de los niños, y más de un $70 \%$ de los adolescentes con obesidad, llegarán a ser adultos obesos, con el impacto negativo para la salud y la carga de enfermedad que conlleva (Bernal, Ponce, Sotomayor, y Carmona, 2012).

El tratamiento de la obesidad no es fácil y en la mayoría de los casos conduce al fracaso. Los resultados obtenidos a largo plazo son desalentadores y muestran que entre el $80 \%$ y $90 \%$ de los niños que han realizado un tratamiento para bajar peso, vuelven a su percentil de peso previo, siendo la tasa de recidiva comparable a la de los adultos (Fernández, 2005). El objetivo principal de cualquier tratamiento de sobrepeso u obesidad es conseguir un peso adecuado a la talla dentro de los parámetros saludables, 
junto a un crecimiento y desarrollo adecuado. El punto clave del tratamiento de la obesidad, se nos antoja pensar que debería ser la alimentación saludable y el aumento de actividad física, pero la simplificación del tratamiento solo puede llevar al fracaso a medio y largo plazo ya que existen variables sociodemográficas, culturales y psicológicas que están incidiendo en el problema y cuya comprensión requiere que se investiguen otros aspectos que puedan incidir en la aparición y mantenimiento del sobrepeso y la obesidad infantil.

Ante este panorama, las futuras líneas de investigación tienen un campo abierto a identificar los factores de riesgo y variables implicadas en el sobrepeso y obesidad infantil, a fin de establecer las medidas convenientes para su control.

Una de las variables que consideramos interesantes explorar dentro de este tema es la percepción de la imagen corporal que los escolares tienen en relación con su peso corporal. Diferentes estudios centrados en la percepción materna del peso de sus hijos concluyen que las madres infravaloran el peso real de sus hijos (Ávila, Gutiérrez, Martínez, y Vázquez, 2017; Flores, Aguado, Cerda, Cortés, y Dávila, 2016; Lara, Flores, Alatorre, Sosa, y Cerda, 2011; Vidarte, Dávila, Castillo, y Díaz, 2017), principalmente si estos presentan sobrepeso u obesidad. Este hecho nos puede hacer pensar que la percepción que tengan los progenitores sobre su hijo puede influir en la autopercepción del niño sobre su peso (Tognarelli, 2012). Así, consideramos importante evaluar qué percepción tiene el niño de su imagen y cómo percibe su peso y su salud, ya que, en el caso de tener que hacer una intervención, este dato será importante a la hora de diseñar intervenciones adaptadas a los menores. Los niños con alteraciones en su estado nutricional, en general, no toman conciencia de su condición, lo que genera un obstáculo para la detección del problema, el abordaje y la resolución del mismo. Como indican Origlia y Zurro (2017), este tipo de trabajos en los que se enfoca la opinión del niño ayudan a fomentar su empoderamiento y así él mismo pueda reconocer sus inquietudes y tomar decisiones hacia su propio bienestar.

La presente investigación tuvo como objetivos: evaluar la relación entre la percepción del estado nutricional de los escolares mediante imágenes y a través de medidas objetivas; evaluar el grado de satisfacción que sobre su imagen corporal tienen los escolares y evaluar la percepción que sobre la salud y enfermedad tienen los escolares de segundo de Primaria.

\section{MÉTODO}

El presente trabajo forma parte de una investigación más amplia sobre la percepción parental del estado nutricional de sus hijos en edad escolar. Para fines de este artículo se presentará la información correspondiente a la percepción de los escolares de segundo de Primaria, la satisfacción con su imagen corporal y la valoración de la salud y 
la enfermedad en relación con el estado nutricional. Este estudio es de tipo cuantitativo, descriptivo, transversal.

El estudio estuvo dirigido a escolares de segundo curso de Educación Primaria, entre 7 y 8 años de varios centros escolares de Logroño elegidos al azar. Usando el programa para análisis epidemiológico de datos tabulados (EPIDAT) versión 3.1 se calculó el tamaño de muestra en 312 a lo cual se le ha agregado un margen de error de $15 \%$, constituyendo una muestra estimada de 359 escolares. Y con la ayuda de una tabla de números aleatorios se eligieron los colegios participantes necesarios para completar la muestra.

Para llevar a cabo el estudio se solicitó autorización a la Consejería de Educación, Formación y Empleo de la Comunidad Autónoma de La Rioja (España) y a las Direcciones y Departamentos de Educación Física de los colegios seleccionados. Así mismo, se solicitó el consentimiento firmado a los padres o tutores legales de los menores que quisieron participar en el estudio, garantizando en todo momento la confidencialidad de los datos y de los participantes. En coordinación con los profesores de Educación Física se estableció un día y hora para que dos miembros del equipo investigador, entrenados previamente para ello, acudieran al centro para hacer las mediciones antropométricas de peso con una balanza digital marca Tanita BC-601 y talla con un estadiómetro portátil desmontable de la marca SECA 217. Se aplicó una breve encuesta de autopercepción corporal basada en el test de imágenes de Warschuburger y Kröller, donde se les pedía a los escolares que seleccionaran la imagen que se correspondía con ellos y la imagen a la que les gustaría parecerse. Utilizando como base las mismas imágenes del test se les preguntó también qué imagen consideraban que estaba sana y qué imagen podía estar enferma. Y para finalizar la encuesta se les preguntaba cómo consideraban ellos que era su salud ofreciéndoles las alternativas de muy buena, buena, regular, mala y muy mala. Después de completar el test se realizaron las mediciones antropométricas: los estudiantes se midieron descalzos, sin prendas ni adornos en la cabeza y sin sudaderas. Con los datos de peso y altura se calculó el Índice de Masa Corporal (IMC) y con el programa WHO Anthro Plus, se calcularon los percentiles de los escolares clasificándolos en delgadez severa (percentil <3), riesgo de delgadez (percentil 4-9), normopeso (percentil 3-84), sobrepeso (percentil 85-94) u obesidad (percentil > 95) según los estándares de la OMS. Los datos se analizaron estadísticamente con el programa SPSS (versión 20), utilizando estadística descriptiva e inferencial.

El estudio se desarrolló siguiendo los principios éticos recogidos en la declaración de Helsinki (1964) y en su última revisión en Seúl (2008). Se solicitó la aprobación del estudio por el Comité de Ética e Investigación de La Rioja (CEICLAR). 


\section{RESULTADOS}

La muestra para el presente estudio estuvo constituida por 374 escolares de ambos sexos, tras eliminar a 5 sujetos por no presentar las respuestas adecuadas para el tratamiento de datos, de los cuales 176 (47\%) fueron niños y 198 (53\%) niñas. Todos ellos eran alumnos que cursaban estudios de segundo de Primaria en 9 centros de la ciudad de Logroño (España). En concreto, 182 (49\%) estudiaban en centros Públicos (representados por 6 de los 9 centros), mientras que 194 (51\%) estudiaban en centros Concertados.

En la tabla 1 se muestran las frecuencias y porcentajes para la variable percentil recodificada en las cinco categorías de percentil de la OMS.

Tabla 1. Percentil de la OMS en categorías

\begin{tabular}{lcc}
\hline Categoría & Frecuencia & Porcentaje \\
\hline Bajo peso & 1 & 0.3 \\
\hline Riesgo de bajo peso & 5 & 1.3 \\
\hline Normopeso & 231 & 61.8 \\
\hline Sobrepeso & 67 & 17.9 \\
\hline Obesidad & 70 & 18.7 \\
\hline Total & 374 & 100 \\
\hline
\end{tabular}

Si nos fijamos en la distribución de percentiles por sexo, encontramos un porcentaje mayor de niños con sobrepeso y obesidad (42.02\%) en comparación con las niñas $(31.73 \%)$.

En relación a la autopercepción de su imagen corporal, el 30.95\% ( $n=113)$ se percibió con bajo peso, un 29.86\% ( $n=109)$ con riesgo de bajo peso, un $37.8 \%(n=138)$ se percibió con normopeso y tan solo un $2.7 \%(n=5)$ se considera con sobrepeso u obesidad.

Se realizó una valoración a nivel descriptivo de la satisfacción por la imagen corporal de los participantes en el estudio. Se consideraron dos categorías: 1: Satisfecho (imagen corporal real= imagen corporal deseada) y 2: no satisfecho (imagen corporal real diferente imagen corporal deseada) y se obtuvo un predominio claro de la categoría de Satisfacción $(n=234 ; 63 \%)$ frente a la no satisfacción $(n=140 ; 37 \%)$. Los resultados obtenidos en cuanto a satisfacción según el sexo nos muestras resultados muy similares, $63.6 \%$ en niños y un $62.4 \%$ en niñas con respecto a la satisfacción con su imagen corporal.

A continuación, se realizó un análisis bivariado del grado de asociación entre la Percepción corporal y el estado nutricional de los participantes según la clasificación de percentil. Se trata, consecuentemente, de dos variables ordinales las cuales constan, cada una con cinco categorías. Para ello se aplicó la prueba Tau-b de Kendall. Para ello se realizó una tabla de contingencia $5 \times 5$ con ambas variables (ver Tabla 2). Los 
resultados mostraron una asociación estadísticamente significativa entre ambas variables $(T=10.141 ; p<.001)$.

Tabla 2. Tabla de contingencia $5 \times 5$ (Percentil en categorías *Percepción por imagen)

\begin{tabular}{|c|c|c|c|c|c|c|c|}
\hline & & & \multicolumn{5}{|c|}{ Percepción por imagen } \\
\hline & & & $\mathrm{BP}$ & RBP & NP & SP & OB \\
\hline \multirow{10}{*}{ Percentil } & \multirow{2}{*}{ BP } & Recuento & 0 & 1 & 0 & 0 & 0 \\
\hline & & $\%$ dentro de $\mathrm{Pc}$ & $0.0 \%$ & $100.0 \%$ & $0.0 \%$ & $0.0 \%$ & $0.0 \%$ \\
\hline & \multirow{2}{*}{ RBP } & Recuento & 3 & 1 & 1 & 0 & 0 \\
\hline & & $\%$ dentro de $\mathrm{Pc}$ & $60.0 \%$ & $20.0 \%$ & $20.0 \%$ & $0.0 \%$ & $0.0 \%$ \\
\hline & \multirow{2}{*}{ NP } & Recuento & 97 & 82 & 51 & 0 & 1 \\
\hline & & $\%$ dentro de $\mathrm{Pc}$ & $42.0 \%$ & $35.5 \%$ & $22.1 \%$ & $0.0 \%$ & $0.4 \%$ \\
\hline & \multirow{2}{*}{ SP } & Recuento & 14 & 15 & 38 & 0 & 0 \\
\hline & & $\%$ dentro de $\mathrm{Pc}$ & $20.9 \%$ & $22.4 \%$ & $56.7 \%$ & $0.0 \%$ & $0.0 \%$ \\
\hline & \multirow{2}{*}{ OB } & Recuento & 3 & 13 & 50 & 3 & 1 \\
\hline & & $\%$ dentro de $\mathrm{Pc}$ & $4.3 \%$ & $18.6 \%$ & $71.4 \%$ & $4.3 \%$ & $1.4 \%$ \\
\hline \multirow{2}{*}{ Total } & & Recuento & 117 & 112 & 140 & 3 & 2 \\
\hline & & $\%$ dentro de $\mathrm{Pc}$ & $31.3 \%$ & $29.9 \%$ & $37.4 \%$ & $0.8 \%$ & $0.5 \%$ \\
\hline
\end{tabular}

NOTA: BP: Bajo peso; RBP; Riesgo de bajo peso; NP: Normopeso; SP: Sobrepeso; OB: Obesidad.

Para el análisis de la variable Satisfacción con la imagen corporal se siguió el mismo procedimiento que el enunciado anterior. En este caso se realizó una tabla de contingencia $5 \times 2$ mostrando los resultados una asociación estadísticamente significativa entre ambas variables $(T=-4.623 ; p<.001)$ dándose una relación inversa entre en valor del percentil y el grado de satisfacción.

Se realizaron pruebas de contraste entre sexos para verificar la posible existencia de diferencias en la valoración del estado de salud y enfermedad por medio de imágenes. Los resultados obtenidos de los niños y de las niñas mostraron unos resultados prácticamente similares en sus valoraciones para cada una de las siete imágenes correspondientes a "niño enfermo", "niña enferma", "niño sano" y "niña sana". El 88\% de los escolares consideraron como niño/a enfermo/a a las figuras que corresponden la categoría de sobrepeso y obesidad respectivamente. En cuanto a la elección de niño/a sano/a un $50 \%$ se decantó por las figuras que corresponden a las categorías bajo peso, riesgo de bajo peso y normal.

Previamente a la realización de los análisis diferenciales, se verificó el cumplimiento de la normalidad para los sumatorios de las valoraciones mediante Kolmogorov-Smirnov. Dado que no se pudo verificar dicho supuesto en ninguno de los cuatro casos se realizaron pruebas de comparación de muestras independientes mediante la prueba no paramétrica o de distribución libre de U de Mann-Whithney (Tabla 3). 
Tabla 3. Pruebas de Mann-Withney para las valoraciones realizadas por sexo

\begin{tabular}{lccc}
\hline & $\begin{array}{c}\text { Sumatorio } \\
\text { Niño enfermo }\end{array}$ & $\begin{array}{c}\text { Sumatorio } \\
\text { Niña enferma }\end{array}$ & $\begin{array}{c}\text { Sumatorio } \\
\text { Niño sano }\end{array}$ \\
\hline $\mathrm{U}$ de Mann-Whitney & 17416.50 & 17372.00 & 17194.50 \\
\hline $\mathrm{Z}$ & -.009 & -.060 & -.249 \\
\hline$p$ & .993 & .952 & .803 \\
\hline
\end{tabular}

Por último, se realizó una valoración descriptiva de la opinión que los escolares tenían de su estado de salud (ver Tabla 4).

Tabla 4. Percepción de salud en cuatro categorías

\begin{tabular}{lc}
\hline Categoría & Frecuencia \\
\hline Muy bueno & 156 \\
\hline Bueno & 181 \\
\hline Regular & 33 \\
\hline Malo & 4 \\
\hline Total & 374 \\
\hline
\end{tabular}

Los resultados señalaron que la categoría más representada fue la de "Bueno" con 181 representantes (48.4\%), seguida de "Muy bueno" con 156 representantes (41.7\%), agrupando entre ambas categorías un total de 337 participantes (más del 90\% de la muestra. En categoría "Regular" sólo hubo 33 participantes $(8.8 \%)$ y 4 en la categoría "Malo" (1.1\%).

\section{DISCUSIÓN Y CONCLUSIONES}

En la actualidad existe un creciente número de niños con sobrepeso y obesidad, lo cual refleja un problema de salud de primordial importancia sanitaria y social. Debido a las consecuencias negativas sobre el desarrollo integral de los menores, este problema exige la intervención de los profesionales de la salud para investigar e intervenir sobre los diferentes factores de riesgo y variables involucradas (Sánchez y Ruiz, 2015). Bajo este prisma nuestra investigación buscaba evaluar la relación entre la percepción real del estado nutricional de los escolares de 7 y 8 años y la autopercepción de su cuerpo, así como evaluar su satisfacción con su imagen corporal y su opinión sobre su salud y la imagen de salud y enfermedad que tienen.

Al comparar los datos obtenidos en nuestro estudio, en cuanto a la prevalencia del sobrepeso y obesidad, con los datos obtenidos en el informe Aladino realizado en nuestro país en 2015, destaca la disminución del porcentaje de niños y niñas con sobrepeso en un $5.1 \%$ aunque las cifras de obesidad se mantienen similares. También es destacable que se hayan obtenido cifras más altas en sobrepeso y obesidad en el grupo de los niños frente al de las niñas, cuando parece que hay una creencia generalizada de que los niños presentan menor sobrepeso u obesidad que las niñas porque son más activos. 
En cuanto a la coincidencia entre la percepción del peso informado y del peso real, sólo el $14 \%$ de los escolares eligen la figura adecuada a su percentil y la mayoría infravalora su peso eligiendo en su mayoría figuras de categorías inferiores a la suya. Salvo el único escolar que presentó bajo peso que señaló su imagen en la categoría riesgo de bajo peso y uno de los niños con obesidad que se señaló en el rango de obesidad, el resto de escolares tienden a elegir figuras de categorías inferiores. Si nos fijamos en la tendencia de la curva del percentil real y del percentil deseado ambas presentan simetrías opuestas. Mientras que la tendencia de la curva de percentil real se desplaza hacia la derecha, hacía percentiles más altos siendo su media de 73.3, la tendencia del percentil deseado es hacia la izquierda, hacía percentiles de bajo peso y de riesgo de bajo peso en su mayoría (60.8\%). Estos resultados son similares a los encontrados por Oliva et al. (2016). Esta elección puede ser un reflejo del deseo social por las figuras más delgadas que ya desde edades muy tempranas impregna a nuestros menores. En la misma línea que el estudio realizado por Origlia y Zurro (2017) encontramos que los escolares perciben su imagen corporal real como un estado nutricional normal $(37.8 \%)$. Y similar a otros estudios realizados, la cantidad de escolares que eligieron la figura de sobrepeso y obesidad fue mínima (2.7\%) (Origlia y Zurro, 2017; Ortega et al., 2013)

En relación con la satisfacción con su imagen corporal podemos decir que los niños y niñas de entre 7 y 8 años están satisfechos con su imagen corporal siendo estos datos similares en ambos sexos. Si comparamos nuestros resultados con otros estudios que han valorado la satisfacción por la imagen corporal pero en edades superiores, como son los estudios realizados por Pérez y Chuz (2009) y Enriquez y Quintana (2016) se encuentra que esta variable cambia de signo y es la insatisfacción por la imagen corporal lo predominante, siendo mayor en el grupo de las chicas. Estos datos nos hacen sospechar que en algún momento entre la niñez y la adolescencia la valoración de la imagen corporal se modifica pasando a ser más negativa. A su vez, los resultados nos muestran una relación inversa entre el valor del percentil y el grado de satisfacción con su imagen. Así a mayor percentil menor satisfacción con su imagen. Encontrándose coincidencias en esta conclusión con los estudios mencionados anteriormente.

Si bien los niños y niñas a la edad de 7 y 8 años ya diferencian a sus iguales por sexo, este aspecto es simplemente descriptivo. Como hemos encontrado en nuestros resultados, los estereotipos culturales sobre la imagen cultural no se han filtrado en ellos y no diferencian que el concepto salud y enfermedad vaya ligado al sexo. A la hora de elegir la imagen de figura enferma la mayoría reconocen que tener sobrepeso u obesidad no es saludable, pero no consideran que el bajo peso y el riesgo de bajo peso no sea saludable. Este aspecto es interesante para tener en cuenta a la hora de diseñar campañas de fomento de la salud donde se tomen como modelos sujetos con normopeso y no con 
bajo peso o riesgo de bajo peso. De esta forma familiarizaremos a nuestros niños en estereotipos saludables que les sirvan de referencia.

Aunque la mayoría de los escolares perciben su estado de salud como bueno o muy bueno $(90.1 \%)$ de los que lo perciben como regular el $54.5 \%$ tiene sobrepeso u obesidad y de los que lo perciben como malo el 75\% también presenta esas categorías de percentil. Sería interesante en estudios posteriores explorar más ampliamente la percepción del estado de salud de los escolares y valorar si el peso tiene relación directa a como ellos sienten su salud.

Algunas de estas conclusiones pueden servir para diseñar intervenciones que ayuden a los escolares que presentan sobrepeso u obesidad a tomar conciencia de su problema y ayuden a eliminar obstáculos que dificultan el que alcancen un peso saludable. Igualmente creemos necesario hacer partícipe a los niños de los aspectos que confieren a su salud y que aprendan a evaluarla y valorarla, empoderándoles en las decisiones que van a ir tomando en cuanto a hábitos saludables durante su vida.

Este estudio fue financiado por la Fundación Rioja Salud (FRS), España, y cofinanciado por la Unión Europea a través del Programa Operativo del Fondo Europeo de Desarrollo Regional (FEDER-La Rioja), España código 6FRSABC020, "Una manera de hacer Europa".

\section{REFERENCIAS}

Agencia Española de Consumo, Seguridad Alimentaria y Nutrición (2016). Estudio ALADINO 2015: Estudio de Vigilancia del Crecimiento, Alimentación, Actividad Física, Desarrollo Infantil y Obesidad en España. Madrid: AECOSAN.

Ávila, H., Gutiérrez, G., Martínez, M.L., y Vázquez, L. (2017). Determinar la sensibilidad y especificidad de la Percepción Materna del Peso de su descendiente de la frontera norte de Tamaulipas por palabras/por imágenes frente al estándar de oro. Cultura de Cuidados, 21, 47. doi:10.7184/cuid.2017.47.16

Bernal, M.L., Ponce, G., Sotomayor, S.M., y Carmona, B. (2012). Impacto de una intervención educativa en escolares y madres de familia para la disminución de sobrepeso. Enfermería Neurológica, 11(2), 63-67.

Durá, T., y Sánchez-Valverde, F. (2005). Obesidad infantil: ¿un problema de educación individual, familiar o social? Acta Pediátrica Española, 63, 204-207.

Enríquez, R.E., y Quintana, M.R. (2016). Autopercepción de la imagen corporal y prácticas para corregirla en adolescentes de una institución educativa. Anales de la Facultad de Medicina, 77(2), 117-122.

Fernández, M.E. (2005). Manejo práctico del niño obeso y con sobrepeso en pediatría de Atención Primaria. Badajoz: Sociedad de Pediatría de Atención Primaria de Extremadura.

Flores, Y., Aguado, M.E., Cerda, R.M., Cortés, E.I., Dávila, M.I. (2016). Maternal perceptions in child's weight and unrelated children less than 1 year old. Atención Primaria, 48(9), 579-585.

Flores, Y., Trejo, P.M., Ávila, H., Cárdenas, V.M., Ugarte, A., Gallegos, J., y Cerda, R.M. (2014). Predictores de la percepción materna del peso del hijo con sobrepeso-obesidad. Universitas Psychologica, 13(2), 553-563. doi:10.11144/Javeriana.UPSY13-2.ppmp 
Lara, B., Flores, Y., Alatorre, M.A., Sosa, R., y Cerda, D. (2011). Percepción materna de sobrepeso-obesidad infantil y riesgo de salud en Nuevo Lardero, Tamaulipas, México. Salud Publica de México, 53(3), 258-263.

Ministerio de Sanidad, Servicios Sociales e Igualdad (2012). Informe anual del Sistema Nacional de Salud. Madrid: Ministerio de Sanidad, Servicios Sociales e Igualdad.

Muñoz, M., Mazure, R.A., y Culebra, J.M. (2004). Obesidad y Sistema Inmune. Nutrición Hospitalaria, 19, 319-324.

Oliva, Y., Ordóñez, M., Santana, A., Marín, A.D., Andueza, G., y Gómez, I.A. (2016). Concordancia del IMC y la percepción de la imagen corporal en adolescentes de una localidad suburbana de Yucatán. Revista Biomédica, 27, 49-60.

Organización Mundial de la Salud (2016). Obesidad y sobrepeso. Ginebra: OMS.

Origlia, A., y Zurro, F. (2017). Percepción y satisfacción de la imagen corporal según estado nutricional en niños de $5^{\circ}$ y $6^{\circ}$ grado (tesis de pregrado). Argentina: Universidad Nacional de Córdoba.

Ortega, M.A., Zurita, F., Cepero, M., Torres, B., Ortega, M.A., y Torres, J. (2013). La percepción e insatisfacción corporal en el alumnado de Educación Secundaria de la ciudad de Jaén. Revista Investigación en Educación, 11(2), 123-129.

Pérez, J.O., y Chuz, A. (2009). Autopercepción de la imagen corporal y alimentaria en escolares y adolescentes rurales. Revista Española de Nutrición Comunitaria, 15(4), 200-206.

Sánchez, J.J., y Ruiz, A.O. (2015). Relación entre autoestima e imagen corporal en niños con obesidad. Revista Mexicana de Trastornos Alimentarios, 6, 38-44.

Serra, L. (2003). Obesidad infantil y juvenil en España. Resultados del Estudio enKid (19982000). Medicina Clínica, 121(19), 725-732.

Tognarelli, A. (2012) Representación de apego de niños y niñas con obesidad y la respuesta sensible de sus madres. Suma Psicológica UST, 9(2), 57-67.

Vidarte, N.E., Dávila, O., Castillo, Y., y Díaz, C. (2017). Asociación entre la percepción materna del estado nutricional y el estado nutricional real en niños de dos a cinco años que acuden a los consultorios de crecimiento y desarrollo en los establecimientos de Atención Primaria Chiclayo Oeste y José Olaya del Distrito de Chiclayo (tesis doctoral). Lima: Universidad de San Martín de Porres.

Recibido: 8 de febrero de 2019

Recepción Modificaciones: 22 de febrero de 2019

Aceptado: 8 de marzo de 2019 